\title{
ANALYSIS OF THE NON-WISHBONE MECHANISM IN A VEHICLE WHEEL SUSPENSION
}

\author{
Marek Kwietniewsin, Tadeusz BiL \\ Koszalin University of Technology, Faculty of Mechanical Engineering, Koszalin, Poland \\ e-mail:mkwietniewski@op.pl; tadeusz.bil@tu.koszalin.pl
}

\begin{abstract}
A suspension of the MacPherson strut type is one of the most frequently used suspension systems in passenger cars. A compact structure is an advantage of this type of suspension. A change to the steering angle and camber as a result of shock absorption is a disadvantage. These changes have an impact on driving safety. This article presents a new solution of the vehicle suspension including its analysis. This suspension possesses the advantages of the MacPherson strut which is a compact and simple structure. In the suspension proposed, the steering angle change being the result of shock absorption has been totally eliminated.
\end{abstract}

Keywords: wheel suspension, MacPherson, structure, mechanism

\section{Introduction}

Suspension of the MacPherson strut type is currently the most common suspension system used in small and middle sized passenger cars. This suspension was designed by Erle S. MacPherson, and it was used for the very first time in 1949 in the Ford Vedette car. A compact and simple structure is an advantage of this type of suspension. A disadvantage is a change to the camber during its operation. In order to minimize the unintended changes to the position of the wheels, parameters of the steering rods of the wishbone and shock absorber strut need to be properly selected (Mantaras et al., 2004). Considering the complexity of the mechanism, what is required is an access to analytical models for the purpose of optimization in specific applications. An adequate determination of parameters through optimization permits a significant reduction of undesirable changes to the steering angle and camber of the wheel. Optimization can be used while designing a new suspension and to improve the existing one. The design process of a suspension system consists of the following stages: initial processing, analysis, and final processing which ought to be optimization. At the stage of initial processing, suspension systems are modelled as a combination of kinetic elements. At this stage, equations for the analysis are created. The second stage consists in analysis of displacements, velocities and accelerations based on the equations created. This is followed by the final processing stage, i.e. optimization of the system (Lee et al., 2009).

For the purpose of further improvement of the safety and comfort of driving, electronic systems are used (Sung et al., 2013). These systems, however, necessitate the use of computers which are sensitive to interferences. These systems furthermore increase the manufacturing costs of the suspension (Habibi et al., 2008).

The analysis can be performed with the aid of conversions in the Cartesian coordinate system. A classical approach is used in order to obtain the location, velocity and acceleration of selected points of the mechanism. This analysis is supported with numerical calculations and it makes it possible to determine the optimal values of the structural parameters; it improves the designing process and, finally, it improves the quality of the mechanism (Yang and Abdel-Malek, 2007; Garcia de Jalón and Callejo, 2011; Avilé et al., 2008). 


\section{Purpose of the study}

A spherical joint possesses three degrees of freedom (three perpendicular rotary axes that intersect in one point). This joint can be replaced with three linkages with one degree of freedom each (Bil, 2011). By replacing spherical joints with three linkages with one degree of freedom (rotary or sliding ones), a mechanism can be obtained whose mathematical description contains more independent parameters and, what follows from this, more possibilities.

\subsection{Analysis of the positions of the wheel suspension mechanism}

Analysis of characteristic points was conducted with the aid of homogeneous conversions in the form of matrix product through a vector in a three-dimensional space. The analysis below did not take into account any problems related to the geometrical structure of the car body and of the suspension as well as the related possible collisions of the car elements. The suspension analysis did not take into account the occurrence of clearances and flexibility of such elements as the joints of steering rod ends, tires, etc.

The geometric values have no reference to any specific car.

\subsection{MacPherson strut}

The $B X Y Z$ system of coordinates was accepted as a fixed reference frame.

The location of fixed points $\mathrm{A}$ and $\mathrm{B}$ and the initial position of the movable point $F$ were determined. The values of angles in the straight-ahead position were accepted as known values: $\delta_{y}, \varepsilon_{z}, \vartheta_{z}, \eta_{y}$, marked in Fig. 1, and the fixed lengths were accepted of the elements (lever) of the mechanism: $L_{1}, L_{2}, L_{3}, L_{4}, L_{8}, L_{9}$.

The location of the point $C$ in the fixed $B X Y Z$ system of coordinates was determined as the locations of the point $A$ in the form of the vector $\mathbf{A}$ and rotations of the section $A C=L_{1}$ in relation to the point $A$ by angles $\alpha_{z}$ and $\gamma_{x}$, respectively. At the same time, the location of the point $C$ can be determined in relation to the point $B$ as a rotation of the lever $L_{2}$ around the axis $B Z$ of the fixed system of coordinates by the angle $\beta_{z}$.

Therefore, the coordinates were determined of the same point $C$ from two relationships, which had to be identical: $\mathbf{C}_{A}=\mathbf{C}_{B}$. After conversion and comparison of the right hand sides of these equations, a system of three equations with three unknowns was obtained

$$
\begin{aligned}
& -L_{1} \sin \alpha \cos \gamma+A_{x}=L_{2} \cos \beta \\
& L_{1} \cos \alpha \cos \gamma+A_{y}=L_{2} \sin \beta \\
& L_{1} \sin \gamma+A_{z}=0
\end{aligned}
$$

The locations of $D, E, G$ and $H$ points in the $B X Y Z$ fixed system of coordinates were determined in a similar manner with the aid of homogenous conversions.

Knowing the locations of $H$ and $G$ points that were found on the wheel rotary axis, the following dependences could be determined

$$
\eta=a \sin \frac{Y_{H}-Y_{G}}{L_{7}} \quad \vartheta=a \sin \frac{Z_{H}-Z_{G}}{L_{7}}
$$

\subsection{Non-wishbone suspension}

Figure 2 presents a diagram of the suspension.

As compared to the MacPherson strut, no wishbone was used, and the point $C$ of the lower linkage of the suspension with the body was replaced with a cylindrical joint. In the point $A$ of the upper linkage between the suspension and the body, the spherical joint was replaced 


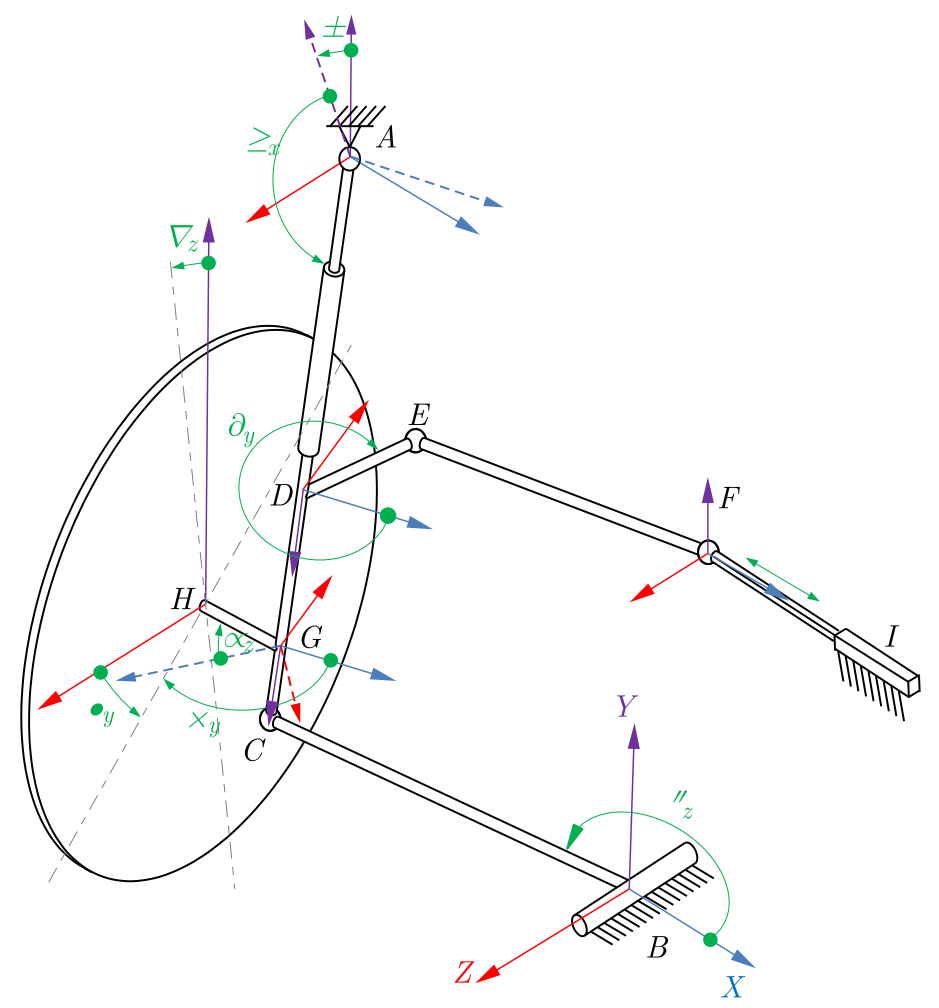

Fig. 1. Diagram of MacPherson suspension; $A$ - centre of the spherical joint of the fixing of the strut to the body, $B$ - centre of the rotary joint of the fixing of the wishbone mechanism to the body, $C$ - centre of the spherical joint of the fixing of the wishbone mechanism and the strut, $D$ - fixed linkage of the

wishbone arm turn and strut, $E$ - spherical joint of the linkage of the steering rod and the strut,

$F$ - spherical joint of the linkage of the steering rod with the steering suspension board,

$G$ - intersection of the wheel axis with the shock absorber axis, $H$ - centre of the wheel, $I$ - steering transmission, $\alpha_{z}$ - lean angle of the shock absorber axis around the immovable $A Z$ axis, $\gamma_{x}-$ lean angle of the shock absorber axis around the movable $A X$ axis, $\delta_{y}$ - camber angle of the wheel axis from the

strut around the $D Y$ axis, $\varepsilon_{z}$ - camber angle of the wheel axis from the strut around the $D Z$ axis, $\vartheta_{z}$ - wheel camber angle, $\eta_{y}$ - wheel steering angle, $\beta_{z}$ - lean angle of the wishbone, $\zeta_{y}$ - lean angle of the arm of the steering rod end from the $D X$ axis, $L_{1}=A C, L_{2}=B C, L_{3}=C D, L_{4}=D E, L_{5}=E F$, $L_{8}=C G, L_{9}=G H-$ linear dimensions of the mechanism

with a revolute joint. Both these changes guarantee the proper steering angle and suspension movement. To provide control of the steering angle and, at the same time, to make the steering angle independent from suspension movement, one of the spherical joints in the point $E$ was replaced with a cylindrical joint with the axis being parallel to the damper axis.

The $A X Y Z$ system of coordinates was accepted as a fixed reference frame.

The initial positions of the movable $C$ and $F$ points were determined in the analysed variant of the suspension.

The values of angles in the straight-ahead position were accepted as known values: $\alpha_{z}, \gamma_{x}$, $\delta_{y}, \varepsilon_{z}, \vartheta_{z}, \eta_{y}$ marked in Fig. 2, and the fixed lengths were accepted of the elements (lever) of the mechanism: $L_{1}, L_{3}, L_{4}, L_{8}, L_{9}$.

The locations of the points $C, D, E_{1}, E_{2}, G$ and $H$ in the $A X Y Z$ fixed system of coordinates were determined with the aid of homogenous conversions.

Using the new locations of the points $G$ and $H$, the angles $\eta_{y}$ and $\vartheta_{z}$ can be determined from Eqs. (2.2). 


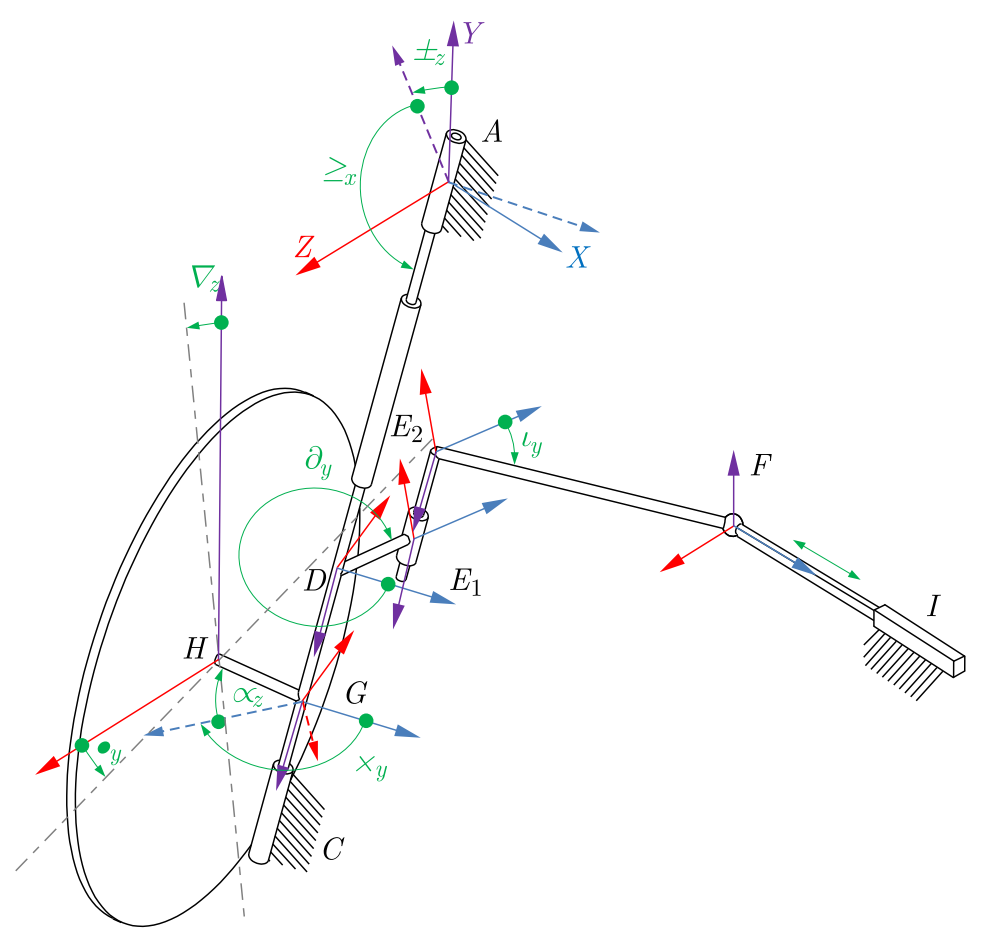

Fig. 2. Non-wishbone vehicle suspension with steering; $A$ - centre of the revolute joint of the upper linkage of the damper with the body, $C$ - centre of the cylindrical joint of the lower linkage of the damper with the body, $D$ - fixed linkage of the steering arm and the damper, $E_{1}-$ cylindrical joint of the linkage of the steering rod with the damper, $E_{2}$ - bending point of the steering rod, $F$ - spherical joint of the linkage of the steering rod with the steering suspension board, $G$ - intersection of the wheel axis with the shock absorber axis, $H$ - centre of the wheel, $I$ - steering transmission, $\alpha_{z}$ - lean angle of the shock absorber axis around the immovable $A Z$ axis, $\gamma_{x}$ - lean angle of the shock absorber around the axis, $\delta_{y}$ - camber angle of the wheel axis from the shock absorber around the $D Y$ axis, $\varepsilon_{z}-$ camber angle of the wheel axis from the shock absorber around the $D Z$ axis, $\iota_{y}$ - rotation of the steering rod around the $E_{1} E_{2}$ axis, $\vartheta_{z}$ - wheel camber angle, $\eta_{y}$ - wheel steering angle, $\zeta_{y}$ - lean angle of the arm of

the steering rod end from the $D X$ axis, $L_{1}=A C, L_{2}=B C, L_{3}=C D, L_{4}=D E, L_{5}=E_{1} E_{2}$, $L_{6}=E_{2} F, L_{8}=C G, L_{9}=G H$ - linear dimensions of the mechanism

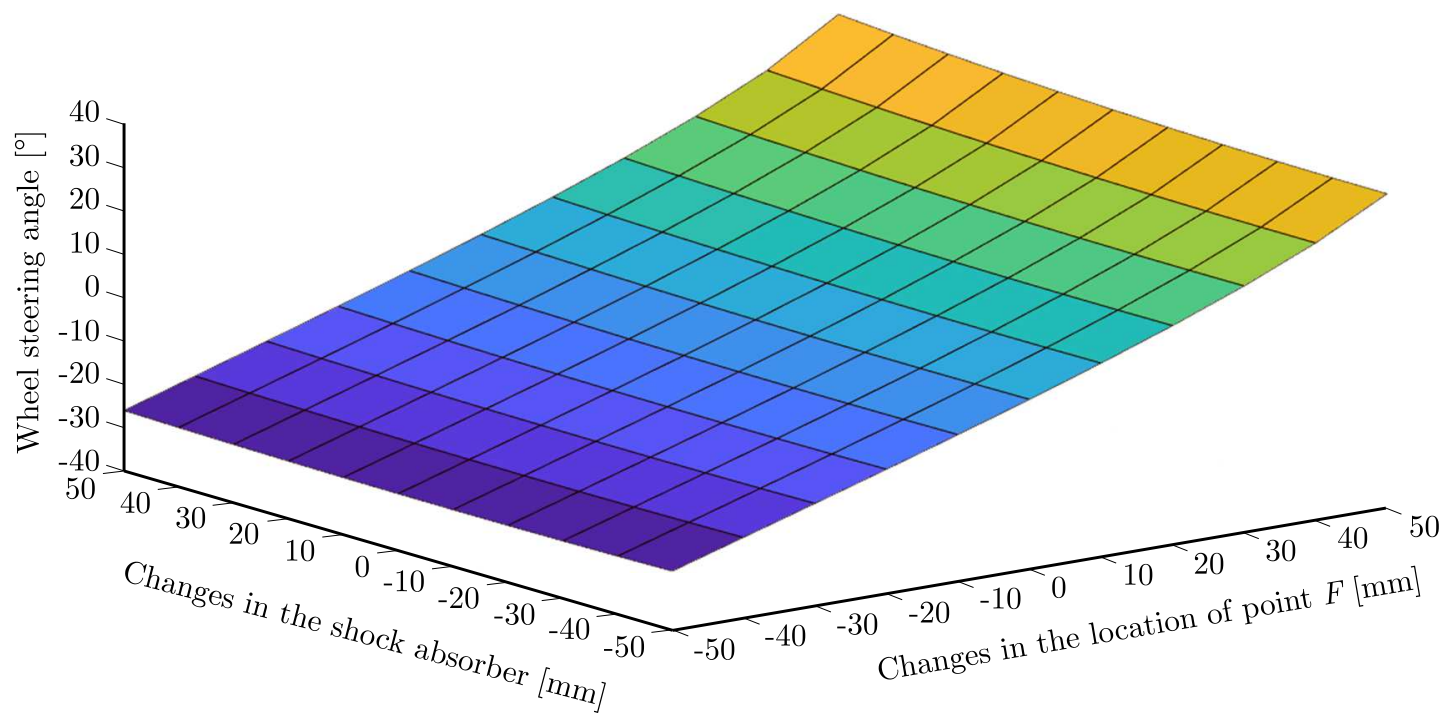

Fig. 3. Diagram of the wheel steering angle depending on the extension of the shock absorber and changes in the location of the point $F$ 


\section{Results}

\subsection{MacPherson strut (Kwietniewski and Bil, 2018)}

The results of calculations of the angles $\eta$ and $\vartheta$ depending on the variable length of the shock absorber $L_{1}$ and a change in the position of the steering wheel are presented in Figs. 3, 4 and 5. The following initial values of the lever lengths, point coordinates and angles were accepted: $L_{1}=600 \mathrm{~mm}, L_{2}=500 \mathrm{~mm}, L_{3}=250 \mathrm{~mm}, L_{4}=110 \mathrm{~mm}, L_{8}=80 \mathrm{~mm}, L_{9}=100 \mathrm{~mm}$, $X A=400 \mathrm{~mm}, Y A=550 \mathrm{~mm}, Z A=20 \mathrm{~mm}, X F=50 \mathrm{~mm}, Y F=200 \mathrm{~mm}, Z F=200 \mathrm{~mm}$, $\delta=179^{\circ}, \varepsilon=10^{\circ}, \zeta=280^{\circ}, \vartheta=1.2^{\circ}, \eta=0.6^{\circ}$.

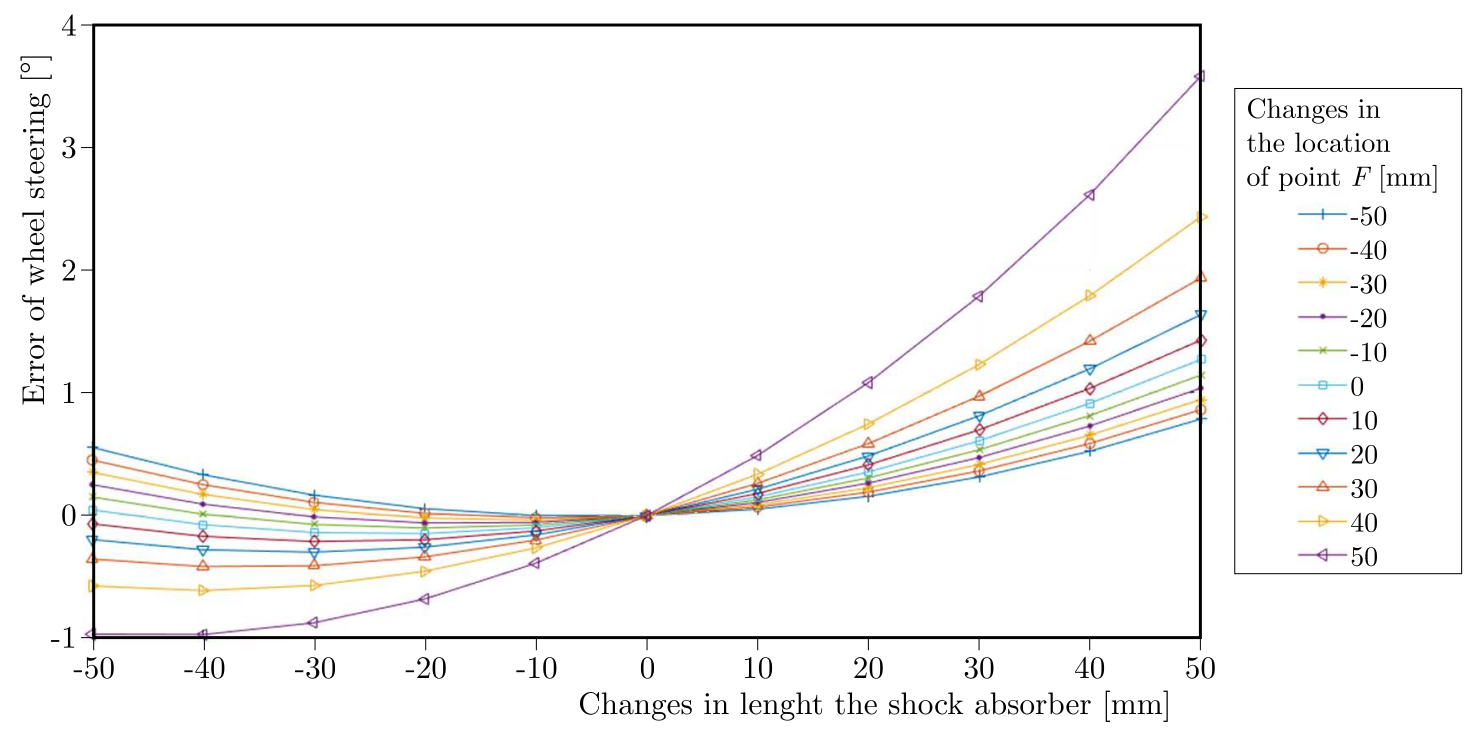

Fig. 4. Diagram of the error of the wheel steering angle depending on the extension of the shock absorber

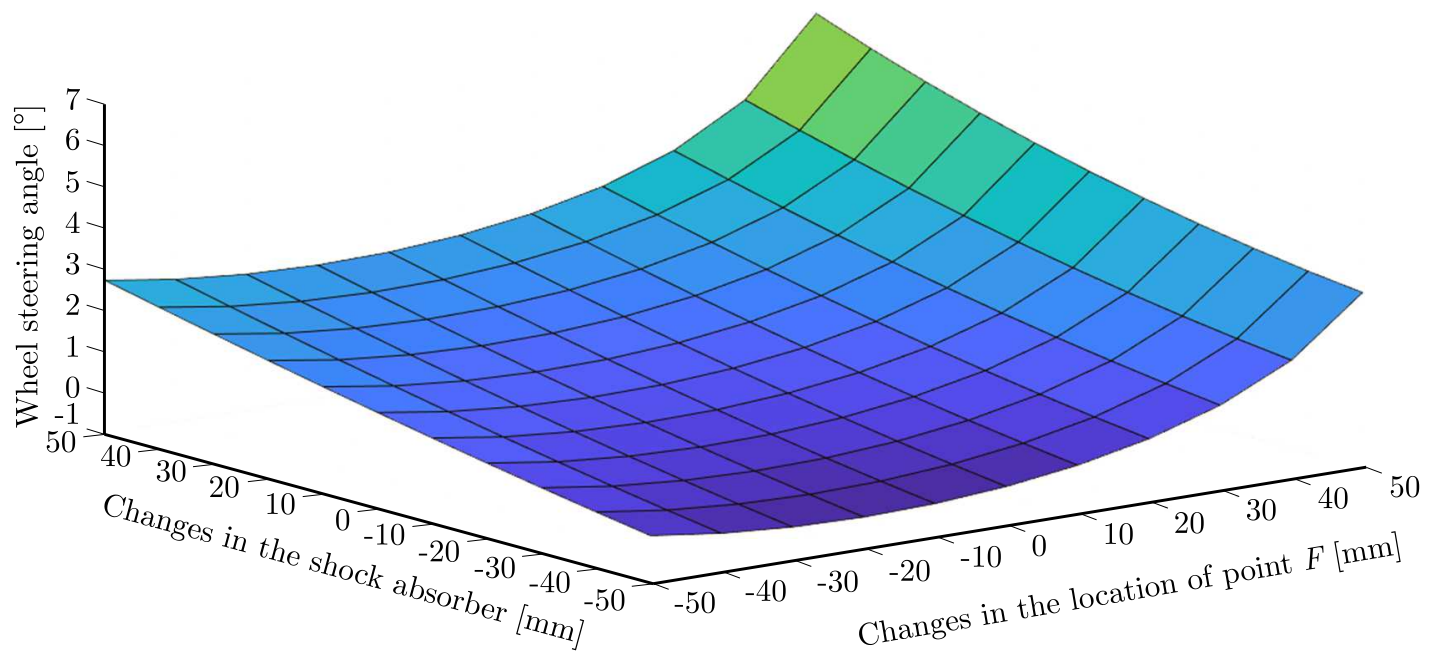

Fig. 5. Diagram of the wheel camber angle depending on the extension of the shock absorber and changes in the location of the point $F$

\subsection{Non-wishbone suspension including a modified steering system}

The results of calculations of the angles $\eta$ and $\vartheta$ depending on the variable length of the shock absorber $L_{1}$ and changes in the position of the steering wheel are presented in Figs. 6, 7 and 8 . 


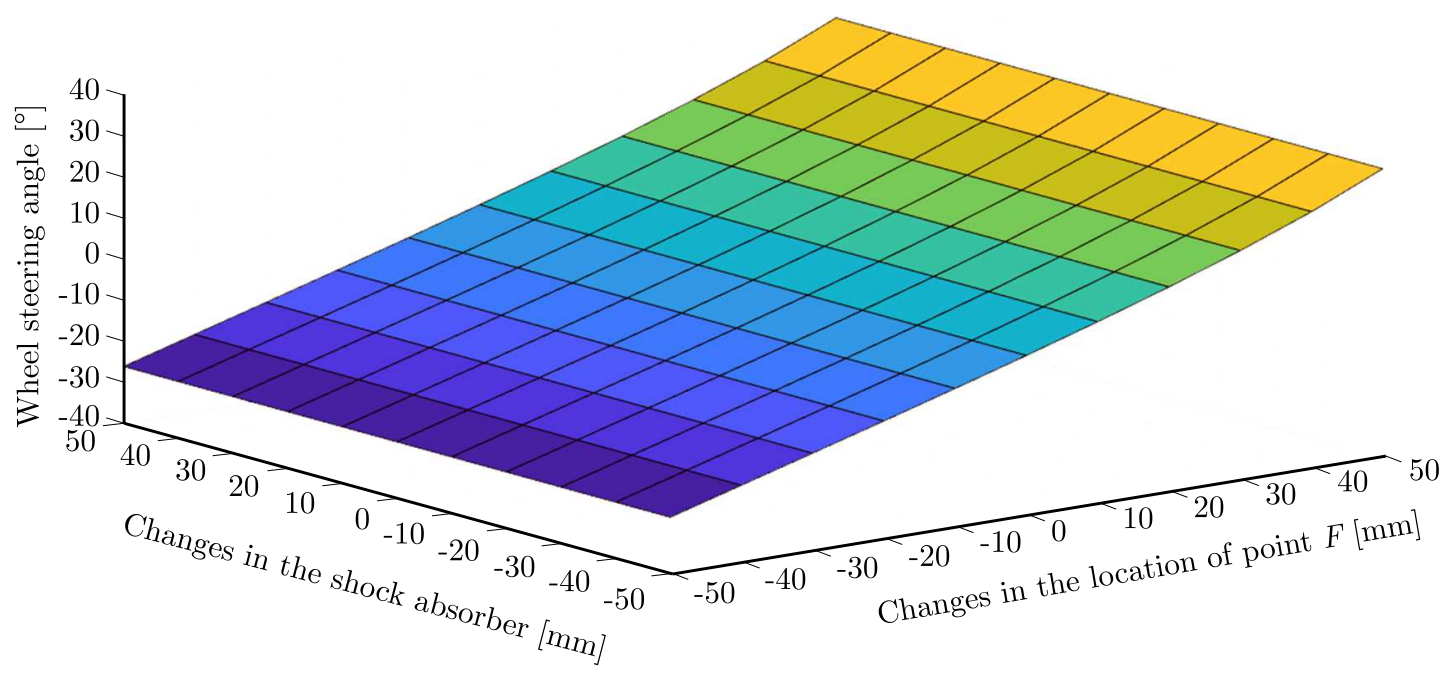

Fig. 6. Diagram of the change of the wheel steering angle depending on the extension of the shock absorber and changes in the location of point $F$

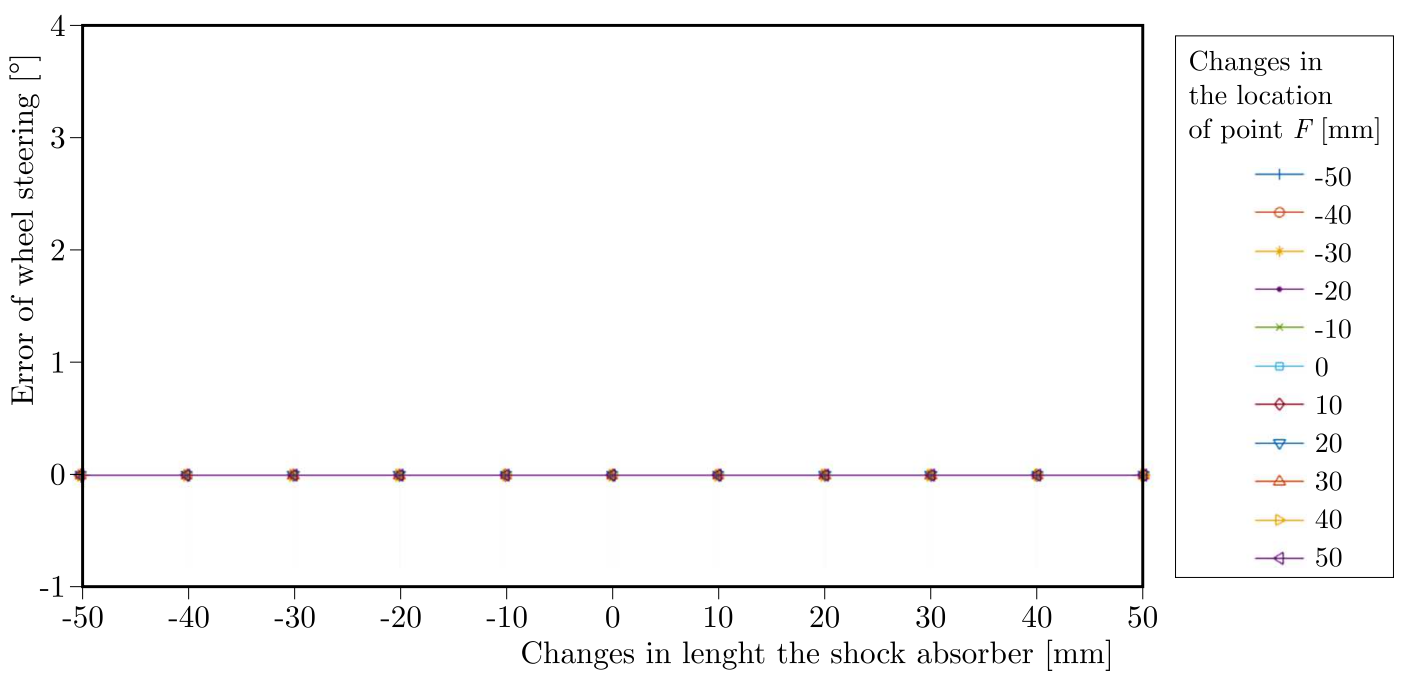

Fig. 7. Diagram of the error of the wheel steering angle depending on the extension of the shock absorber

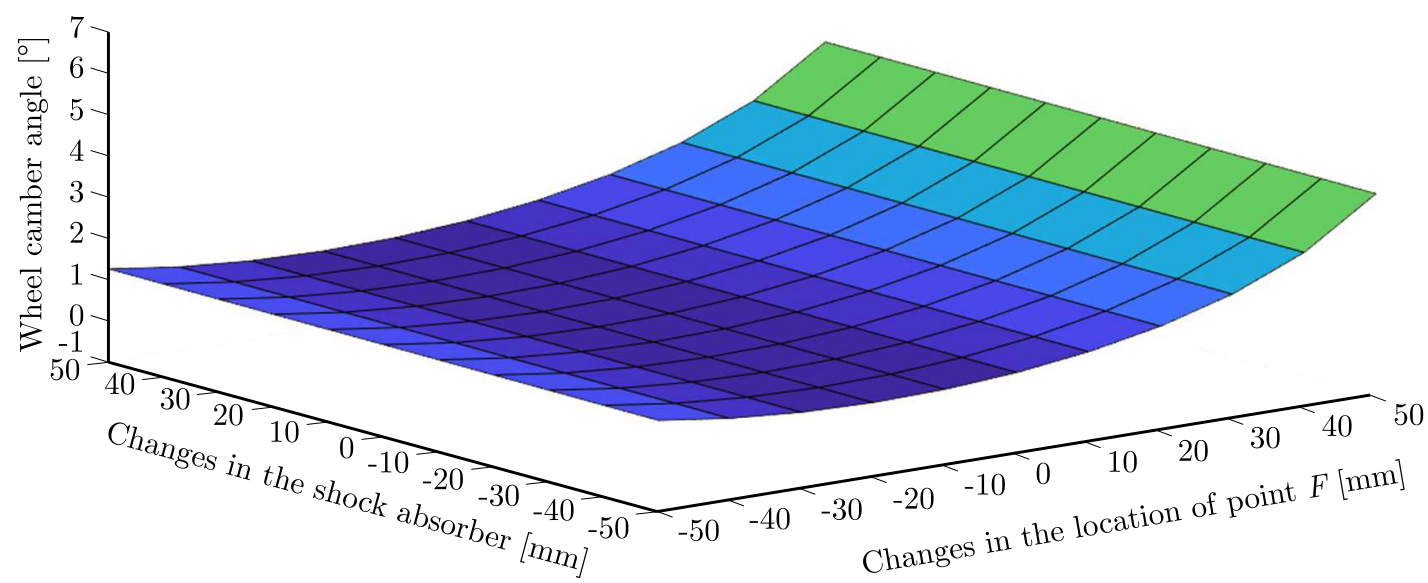

Fig. 8. Diagram of the wheel camber angle depending on the change in the extension of the shock absorber and changes in the location of the point $F$ 
The following initial values of the lever lengths, point coordinates and angles were accepted: $L_{1}=600 \mathrm{~mm}, L_{3}=250 \mathrm{~mm}, L_{4}=110 \mathrm{~mm}, L_{8}=80 \mathrm{~mm}, L_{9}=100 \mathrm{~mm}, X F=450 \mathrm{~mm}$, $Y F=350 \mathrm{~mm}, Z F=180 \mathrm{~mm}, \alpha=9^{\circ}, \gamma=178^{\circ}, \delta=179^{\circ}, \varepsilon=10^{\circ}, \zeta=280^{\circ}, \vartheta=1.2^{\circ}$, $\eta=0.6^{\circ}$.

\section{Conclusions}

The article presents an analysis of the MacPherson suspension mechanism and of a new type of a non-wishbone suspension. It is demonstrated that the operation of this suspension system is prone to be erroneous. As a result of the suspension movement, there occurs an unintended wheel steering angle, which may be followed by a change in the driving direction, not predicted by the driver. In order to eliminate this inconvenience, a new suspension type has been proposed in which the spherical joints are replaced with another type of joints. In the suspension proposed, the impact of the suspension movement on the unintended wheel steering angle is totally eliminated, while the compact structure of the suspension is preserved. In order to obtain independence of the wheel steering angle of the suspension movement, the rotary axis of the steering rod $E 1$ needs to be parallel to the axis of the suspension movement $A C$.

\section{References}

1. Avilés R., Hernández A., Amezua A., Altuzarra O., 2008, Kinematic analysis of linkages based in finite elements and the geometric stiffness matrix, Mechanism and Machine Theory, 43, 964-983, DOI: 10.1016/j.mechmachtheory.2007.07.007

2. BIL T., 2011, Kinematic analysis of a universal spatial mechanism containing a higher pair based on tori, Mechanism and Machine Theory, 46, 412-424

3. Garca de Jalón J., CAllejo A., 2011, A straight methodology to include multibody dynamics in graduate and undergraduate subjects, Mechanism and Machine Theory, 46, 168-182, DOI: 10.1016/j.mechmachtheory.2010.09.008

4. Habibi H., Shirazi K.H., Shishesaz M., 2008, Roll steer minimization of McPherson-strut suspension system using genetic algorithm method, Mechanism and Machine Theory, 43, 57-67

5. Kwietniewski M., Bil T., 2018, Analysis of a modified design of MacPherson column (in Polish), Autobusy, 11, 41-44, DOI: 10.24136/atest.2018.344

6. Lee H.G., Won C.J., Kim J.W., 2009, Design sensitivity analysis and optimization of McPherson suspension systems, Proceedings of the World Congress on Engineering, WCE, II

7. Mantaras D.A., Luque P., Vera C., 2004, Development and validation of a three-dimensional kinematic model for the McPherson steering and suspension mechanisms, Mechanism and Machine Theory, 39, 603-619

8. Sung K.-G., Seong M.-S., Choi S.-B., 2013, Performance evaluation of electronic control suspension featuring vehicle ER dampers, Meccanica, 48, 121-134

9. Yang J., Abdel-Malek K., 2007, Design propagation in kinematics of mechanical systems, Mechanism and Machine Theory, 42, 807-824 